\title{
The Intrahepatic Expression and Distribution of BTLA and its Ligand HVEM in patients with HBV-related acute-on-chronic liver failure
}

\author{
Huan $\mathrm{Xu}^{1,2+}$, Dayan $\mathrm{CaO}^{1,2+}$, Guoning Guo ${ }^{3}$, Zhihua Ruan ${ }^{4}$, Yuzhang $\mathrm{Wu}^{2}$ and Yongwen Chen ${ }^{1,2^{*}}$
}

\begin{abstract}
Objective: It has been demonstrated that signals from the inhibitory receptor B and T lymphocyte attenuator (BTLA) are involved in regulating the pathogenesis of infectious diseases. However, the expression and anatomical distribution of BTLA and its ligand, the herpes virus entry mediator (HVEM), have not yet been determined in cases of HBV-related acute-on-chronic liver failure (HBV-ACLF) patients.
\end{abstract}

Methods: In this study, the expression of BTLA and HVEM in liver tissues from HBV-ACLF, chronic hepatitis B (CHB) patients and healthy individuals was analyzed by immunohistochemistry.

Results: The results of this analysis demonstrated that both molecules were observed in the HBV-ACLF samples and that their expression was chiefly in the infiltrating inflammatory cells and the damaged bile ducts. However, they were absent in liver sections from CHB patients and healthy controls. Immunofluorescence double-staining indicated that BTLA was found on CK-18 ${ }^{+}$epithelial cells, CD31 $1^{+}$endothelial cells, CD68 ${ }^{+}$macrophages, CD56 ${ }^{+} \mathrm{NK}$ cells, CD $16^{+}$monocytes, $\mathrm{CD}^{+}, \mathrm{CD}^{+}{ }^{+}$cells, and Foxp3 ${ }^{+}$regulatory T cells (Treg). By contrast, HVEM expression was restricted to $\mathrm{CK}_{1} 8^{+}$epithelial cells and $\mathrm{CD} 8^{+}$macrophages. Moreover, the expression of several members of the $\mathrm{B} 7$ superfamily, including PD-L1, PD-L2, B7-H3 and B7-H4, was also detected in these liver tissues, and these proteins were co-expressed with HVEM. Interestingly, the expression of fibrinogen-like protein 2 (FGL2), a virus-induced procoagulant molecule, was also found in liver sections from HBV-ACLF, this molecule also co-expresses with BTLA and HVEM.

Conclusions: These results suggest that BTLA-HVEM signaling is likely to affect the pathogenesis of HBV-ACLF, a clear understanding of the functional roles of these proteins should further elucidate the disease process.

Virtual slides: The virtual slide(s) for this article can be found here: http://www.diagnosticpathology.diagnomx.eu/ vs/8080806838149123

Keywords: BTLA, HBV-ACLF, HVEM, Immunohistochemistry, B7 superfamily

\section{Introduction}

Hepatitis B virus (HBV) infection causes a spectrum of diseases, such as chronic hepatitis B (CHB), liver cirrhosis, primary hepatocellular carcinoma, and acute-on-chronic liver failure (ACLF) [1]. HBV-ACLF is a clinical syndrome that is defined as acute hepatic insult with either diagnosed

\footnotetext{
* Correspondence: yongwench@163.com

${ }^{\dagger}$ Equal contributors

'Institute of Immunology, PLA, Third Military Medical University, Chongqing

400038, People's Republic of China

${ }^{2}$ Undergraduate Administration Office, Third Military Medical University,

Chongqing 400038, People's Republic of China

Full list of author information is available at the end of the article
}

or undiagnosed chronic liver disease [2,3]. The hallmark of HBV-ACLF is the extreme rapidity of the necromicroinflammatory process, which results in hepatocellular necrosis within several weeks [4]. Currently, liver transplantation is the best method for clinical treatment of this disease. However, the mortality of HBV-ACLF remains very high. It is essential to identify more specific markers for the diagnosis of this disease.

$\mathrm{B}$ and T lymphocyte attenuator (BTLA) is a member of the CD28 superfamily [5]. BTLA-deficient $\left(\mathrm{BTLA}^{-/-}\right)$ mice are more sensitive to experimental autoimmune encephalomyelitis (EAE) [5], allergic airway inflammation

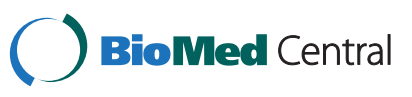


[6], autoimmune hepatitis [7] and MHC-mismatched allograft rejection [8] than normal individuals, indicating that this receptor plays an essential role in maintaining peripheral tolerance. In infectious disease models, for instance, it has been found that an interruption of BTLA signaling causes the augmentation of the virus-specific cytotoxic lymphocyte (CTL) response, promoting the early clearance of both Listeria bacteria and CMV infection $[9,10]$. These studies generally indicate that BTLA appears to be a negative regulator. Herpes virus entry mediator (HVEM), an immunoregulatory molecule that belongs to the tumor necrosis factor (TNF) receptor superfamily, has been identified as the potential 'ligand' for BTLA [11]. The cross-linking of BTLA with HVEM results in the phosphorylation of the tyrosines on BTLA and the recruitment of phosphatases, including SHP-1 and SHP-2, to the ITIM motif in the cytoplasmic region of BTLA, this response reduces TCR signaling and eventually diminishes $\mathrm{T}$ cell activation [12].

Our previous work has demonstrated that BTLA actively participates in the pathogenesis of fulminant hepatitis that is caused by infection with murine hepatitis virus strain 3 (MHV-3) [13]. However, uncertainty remains regarding the expression and anatomical distribution of BTLA and HVEM in livers from HBV-ACLF patients. In this study, we used immunohistochemistry to analyze the expression of both proteins in liver biopsies from HBV-ACLF patients and further assessed the phenotypes via immunofluorescence double staining.

\section{Materials and methods Patients}

Four samples from autopsies of HBV-ACLF patients, 16 samples from liver biopsies of $\mathrm{CHB}$ patients, and 5 samples of liver tissue from normal individuals were used in this study. All of the CHB patients were seropositive for hepatitis B surface Ag (HBs) and hepatitis B e Ag (HBe) for at least 12 months and were negative for antibodies against the human immunodeficiency virus, hepatitis $\mathrm{C}$ virus, and hepatitis $\mathrm{D}$ virus, additional parameters for these $\mathrm{CHB}$ patients have been reported in our previous work $[14,15]$. All of the patients were admitted to the Center of Infectious Disease, Southwest Hospital (Chongqing 400038, People's Republic of China) from June 2006 to January 2009. None of the CHB patients were treated for chronic HBV infection for 6 months prior to the liver sampling process. Sections of liver tissue were scored in a blinded fashion to measure their histological diagnoses. The study protocol was approved by the Ethics Committee of the Third Military Medical University review board.

\section{Immunohistochemistry}

The protocol that was used for immunohistochemistry was in accordance with previously published procedures, with slight modifications [14]. Briefly, paraffin-embedded tissue blocks were cut into $2 \sim 3 \mu \mathrm{m}$ sections and mounted on poly-L-lysine-charged glass slides. After the sections were dewaxed and rehydrated, antigen retrieval was performed by microwaving these sections in $10 \mathrm{mM}$ citrate buffer $(\mathrm{pH}$ 6.0). The sections were cooled to room temperature (RT), and the endogenous peroxidase was blocked by incubation with a solution of $0.5 \%$ hydrogen peroxide $\left(\mathrm{H}_{2} \mathrm{O}_{2}\right)$ in $50 \%$ methanol for $1 \mathrm{~h}$. The sections were then incubated in 3\% BSA plus $0.1 \%$ Nonidet P-40 in PBS for $1 \mathrm{~h}$ at RT to block nonspecific binding. The sections were then incubated overnight at $4^{\circ} \mathrm{C}$ with primary anti-BTLA (1:100, rabbit IgG; Santa Cruz, San Diego, CA, USA), anti-HBs (1:100, rabbit IgG; Abcam, Cambridge, MA, USA), anti-CD68 (1:100, clone: 3F103; Santa Cruz), anti-CD16 (1:100, clone: 2Q1240; Santa Cruz), anti-CD3 (1:50, clone: F7.2.38; Dako, Copenhagen, Denmark) or anti-fibrinogen (1:100; Dako) antibodies that had been diluted in $1 \%$ BSA. After the sections were washed, they were incubated with the corresponding secondary antibodies for $1 \mathrm{~h}$ at RT. The Vectastain ABC kit (Vector Laboratories, Burlingame, CA, USA) was used to implement the avidin-biotin complex approach in accordance with the manufacturer's instructions. Sections that were incubated with isotypematched, concentration-matched immunoglobulin without primary antibodies were used as isotype controls. Peroxidase activity was visualized with the DAB Elite kit (K3465, Dako), and the brown coloration of tissues represented positive staining. The sections were lightly counterstained with hematoxylin, dehydrated through an ethanol series to xylene, and mounted. Finally, the sample sections were viewed using a light microscope (Zeiss Axioplan 2, Berlin, Germany).

\section{Immunofluorescence double staining}

For immunofluorescence double staining, sections were incubated with primary anti-BTLA and anti-HVEM antibodies at $4^{\circ} \mathrm{C}$ overnight. After the sections were washed with PBS (5 min/wash $\times 3$ washes), they were incubated with Alexa Fluor ${ }^{\circledR}$ 555-conjugated goat anti-mouse/rabbit IgG antibodies (Invitrogen, San Diego, CA, USA) for $1 \mathrm{~h}$. Subsequently, the sections were further incubated with anti-CD3, anti-CD8 (1:50, clone: C8/144B; Dako, Denmark), anti-CD56 (1:50; Santa Cruz), anti-B7-H1, (1:100, clone: 29E.2A3; kindly provided by Dr. Gordon J. Freeman (Department of Medical Oncology, Dana-Farber Cancer Institute, Harvard Medical School, USA)), anti-B7-DC (either 1:50, polyclonal goat IgG; R\&D Systems or 1:100, clone: 24F.7G12; kindly provided by Dr. Gordon J Freeman), anti-PD-1 (2 $\mu \mathrm{g} / \mathrm{ml}$, AF1086; R\&D Systems), anti-CD68, anti-FGL2 (1:100, clone: 4H5; Santa 
Cruz), anti-CD31 (1: 50; Santa Cruz), anti-CK-18 (1: 200; Santa Cruz), or anti-FGL2 (1:100; Santa Cruz) antibodies at $4^{\circ} \mathrm{C}$ overnight and incubated with Alexa Fluor ${ }^{\circledR}$ 488conjugated goat anti-mouse/rabbit IgG1 antibodies (Invitrogen) for an additional $1 \mathrm{~h}$. Finally, the sections were incubated with $1 \mu \mathrm{g} / \mathrm{ml}$ DAPI (Sigma, St. Louis, MO, USA) for $10 \mathrm{~min}$ to stain the nuclei. Sections incubated with the appropriate isotype control primary antibodies and fluorescently labeled secondary antibodies were used as negative controls. The results were analyzed using fluorescence microscopy (Zeiss Axioplan 2).

\section{Results}

The pathologic characteristics of liver tissue from HBV-ACLF

To address the relevance of BTLA and HVEM expression in human viral hepatitis, four liver biopsies from patients with biochemical, histological, and clinical evidence of HBV-ACLF, sixteen cases of $\mathrm{CHB}$ patients and five cases of healthy individuals were collected in this study. Severe hepatocyte necrosis, ballooning degeneration, and collapse of the parenchyma were found in the liver tissue from HBV-ACLF patients, as demonstrated by $H \& E$ staining (Figure $1 \mathrm{~A})$. In parallel, a cohort of $\mathrm{HBs}^{+}$hepatocytes (Figure 1B), infiltrating $\mathrm{CD}^{+} 8^{+}$macrophages (Figure 1C), $\mathrm{CD}^{+} \mathrm{T}$ cells (Figure 1D), CD16+ monocytes (Figure 1E) and a high level of fibrinogen deposited in the damaged hepatocytes (Figure 1F) were also detected in these samples. Based on the histological and immunophenotypic findings from the examined samples, we concluded that these four cases were accurately diagnosed as typical instances of ACLF.
The expression and anatomical distribution of BTLA in sections from HBV-ACLF

The expression of BTLA in liver samples from HBVACLF patients, $\mathrm{CHB}$ patients and healthy individuals was detected by immunohistochemistry. The results showed that BTLA was absent in normal liver tissues (Figure 2A) and in the 16 samples from $\mathrm{CHB}$ patients (Figure 2B). In addition, no positive staining was observed in the sections incubated with rabbit IgG1 isotype antibodies (data not shown). Nevertheless, strongly $\mathrm{BTLA}^{+}$cells were observed in the benign bile ducts (Figure 2C) and in the infiltrating inflammatory cells (Figure 2D) of these four cases of HBV-ACLF.

The phenotypes of $\mathrm{BTLA}^{+}$cells in liver tissues from HBV-ACLF patients were further examined by immunofluorescence double staining. From these analyses, the expression of BTLA was observed on CK- $18^{+}$epithelial cells, $\mathrm{CD}^{+} 1^{+}$endothelial cells, $\mathrm{CD} 68^{+}$macrophages, $\mathrm{CD} 6^{+} \mathrm{NK}$ cells, $\mathrm{CD} 16^{+}$monocytes, $\mathrm{CD}^{+} \mathrm{CD}^{+} \mathrm{T}$ cells, and Foxp $3^{+}$regulatory $\mathrm{T}$ cells (Treg), but this expression was completely absent on PD- ${ }^{+}$cells (Figure 3 ).

\section{The expression and distribution of HVEM in sections from HBV-ACLF}

The expression of HVEM in these collected liver samples was detected by immunofluorescence staining, which indicated that HVEM-positive cells were observed in all of the liver samples from HBV-ACLF patients. This protein was found on cell membranes and in the cytoplasm, and its expression was chiefly found in damaged bile ducts (Figure 4B) as well as in certain infiltrating inflammatory cells (Figure 4C), which were distributed

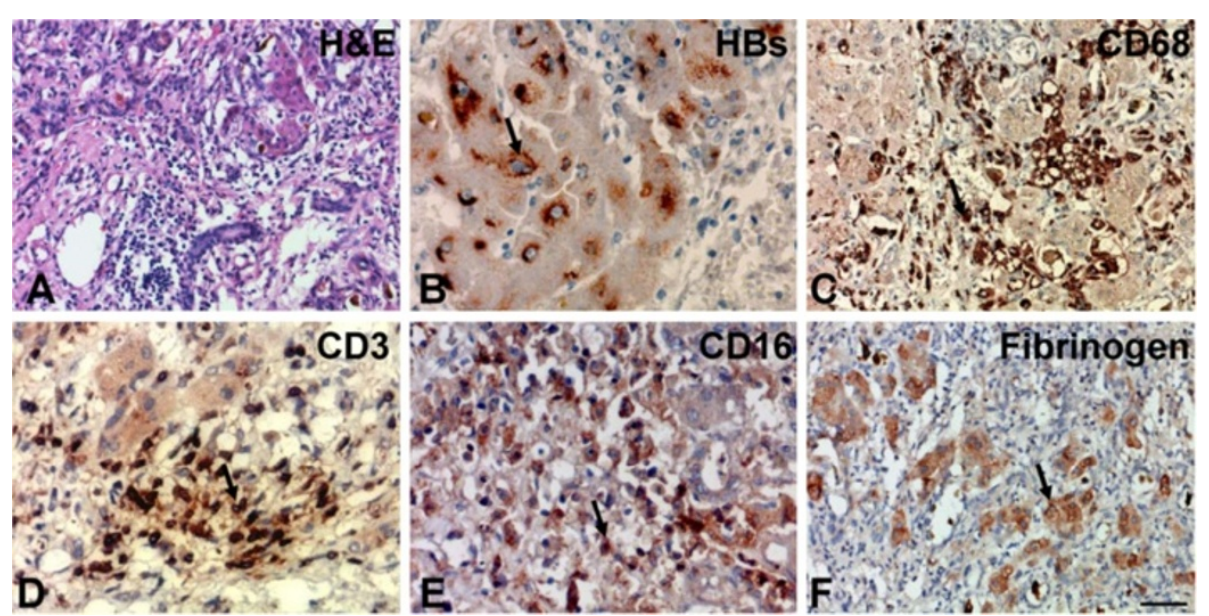

Figure 1 The morphology of liver tissue from patients with HBV-related acute-on-chronic liver failure (HBV-ACLF). (A) HE staining demonstrated that liver tissue sections from HBV-ACLF patients have severe necrosis, hepatocytes with ballooning degeneration and collapse of the parenchyma; immunohistochemistry showed that (B) the expression of HBsAg was found in hepatocytes of HBV-ACLF patients and that a cohort of $(\mathbf{C}) \mathrm{CD}^{+} 8^{+}$macrophages, (D) $\mathrm{CD}^{+} \mathrm{T}$ cells, and (E) $\mathrm{CD} 16^{+}$monocytes and (F) fibrinogen ${ }^{+}$cells were infiltrated and distributed throughout the livers of HBV-ACLF patients. The arrows indicate positive cells, and the scale bar indicates $20 \mu \mathrm{m}$. 


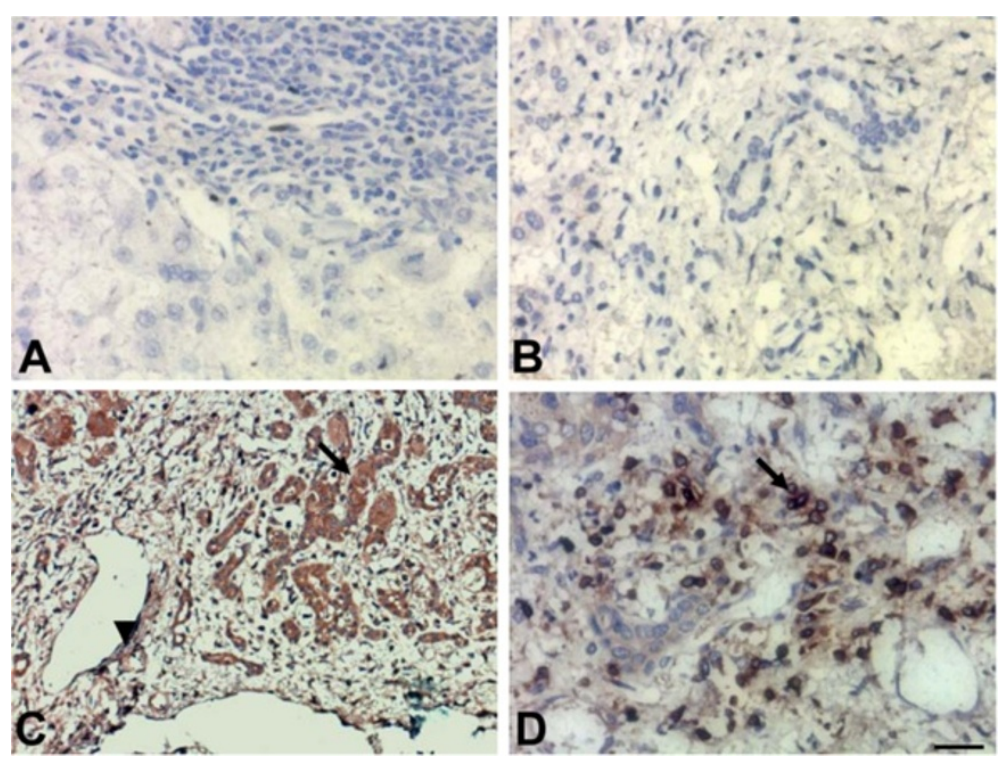

Figure 2 The expression of BTLA in liver tissues from HBV-related acute-on-chronic liver failure (HBV-ACLF) patients, as detected by immunohistochemistry. (A) BTLA expression was absent in normal liver sections; (B) BTLA expression was absent in liver sections from CHB; (C) strong BTLA staining was observed in benign bile ducts sections from HBV-ACLF patients; (D) strong BTLA staining was observed on infiltrating lymphocytes of sections from HBV-ACLF patients. The arrows indicate infiltrating positive cells, arrowhead indicated the capillaries. The scale bar indicates $20 \mu \mathrm{m}$.
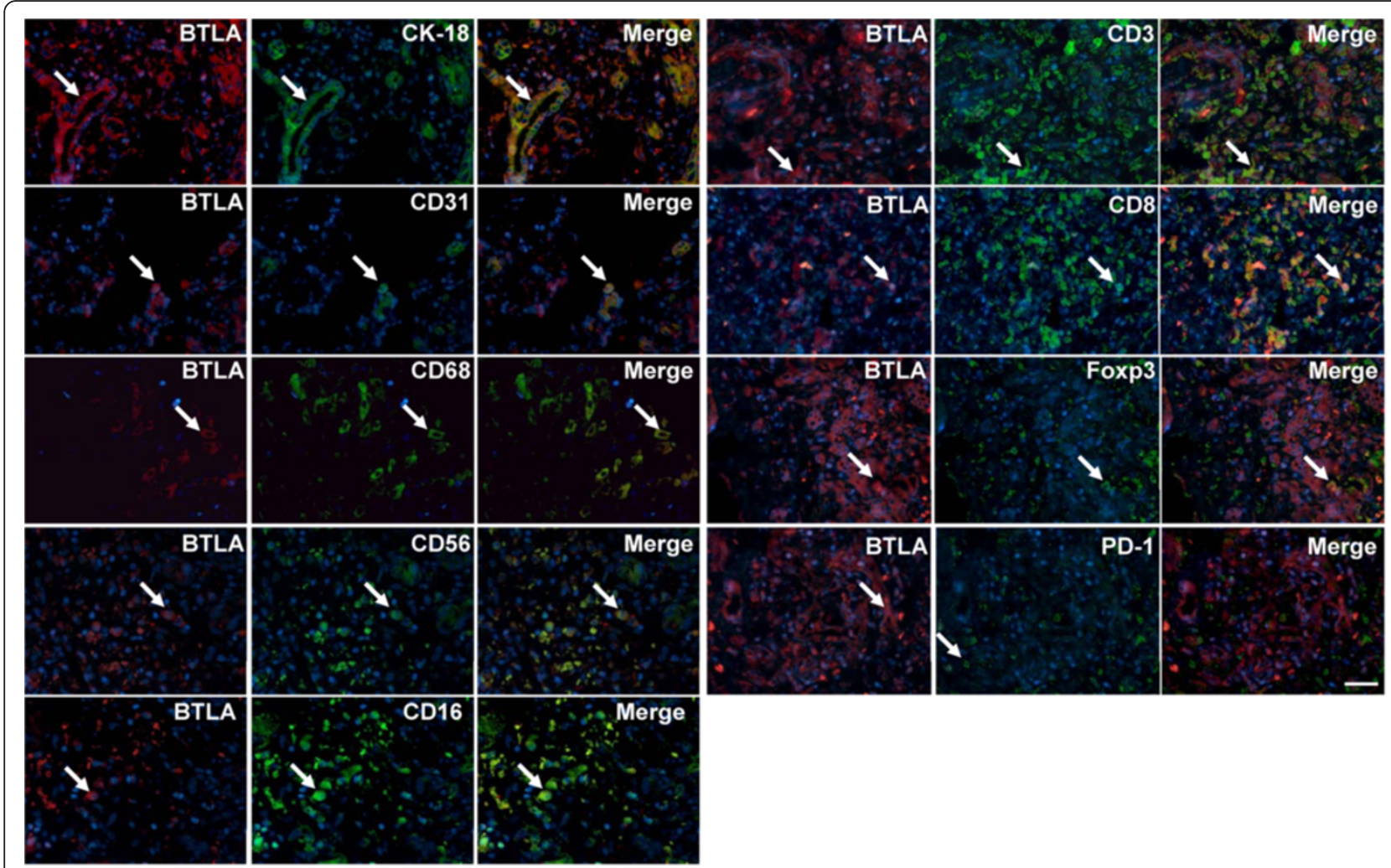

Figure 3 The morphology of BTLA ${ }^{+}$cells in liver tissues from patients with HBV-related acute-on-chronic liver failure (HBV-ACLF) was detected by immunofluorescence double staining. Immunofluorescence double staining revealed that BTLA was expressed on CK-18 ${ }^{+}$epithelial cells, CD $31^{+}$endothelial cells, CD68 ${ }^{+}$macrophages, CD56 $6^{+} \mathrm{NK}$ cells, CD16 ${ }^{+}$monocytes, CD3 ${ }^{+} \mathrm{CD} 8^{+} \mathrm{T}$ cells, and Foxp3 ${ }^{+}$regulatory T cells (Treg), whereas BLTA was absent on PD-1 ${ }^{+}$cells. The arrow indicates positive cells. The nuclei were stained with DAPI, and the scale bar indicates $20 \mu \mathrm{m}$. 


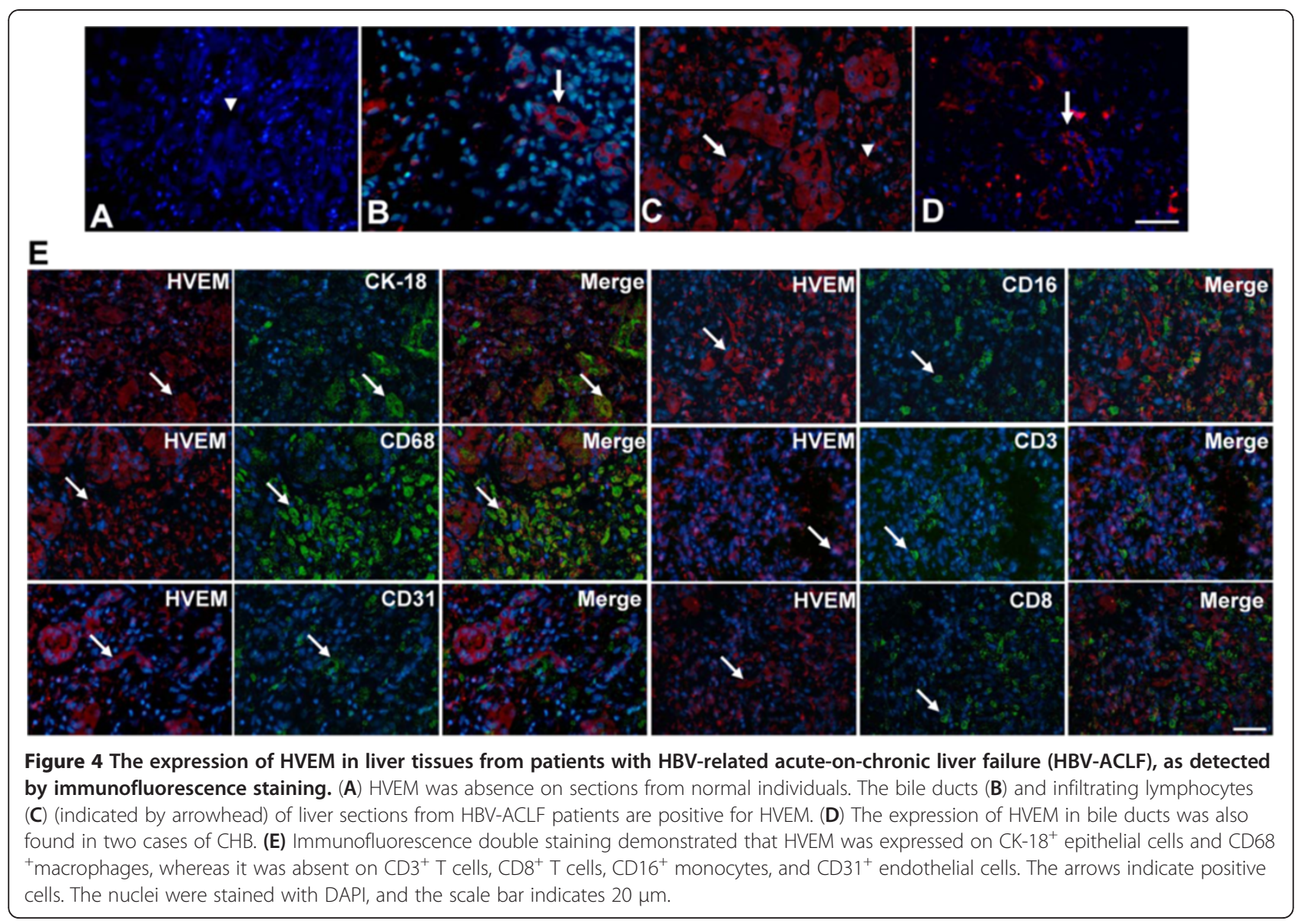

throughout the entire tissue section. In this study, two cases $(2 / 16)$ of $\mathrm{CHB}$ were positive for HVEM, and the expression of HVEM in these instances was restricted to the damaged bile ducts (Figure 4D). However, normal liver tissues were negative for HVEM (Figure 4A). These results indicate that the expression of HVEM was induced in liver samples from HBV-ACLF patients.

The phenotypes of $\mathrm{HVEM}^{+}$-cells within HBV-ACLF liver sections were further examined by immunofluorescence double staining. The results showed that the expression of HVEM was found on $\mathrm{CK}-18^{+}$epithelial cells and $\mathrm{CD} 8^{+}$macrophages, but this expression was absent on $\mathrm{CD} 1^{+}$endothelial cells, $\mathrm{CD} 16^{+}$monocytes, and $\mathrm{CD}^{+}$ $\mathrm{CD}^{+} \mathrm{T}$ cells (Figure 4E).

Our previous work demonstrated that the expression of several members of the $\mathrm{B} 7$ superfamily, including PD-L1 (B7-H1), PD-L2 (B7-DC), B7-H3 and B7-H4, was also detected in liver biopsies from HBV-ACLF patients [14,15]. In the current study, we examined the relationships between HVEM and these members of the B7 superfamily, and the results of this analysis indicated that HVEM was co-expressed with all of these molecules in liver sections from HBV-ACLF patients (Figure 5).
The relationships between BTLA-HVEM and FGL2 in sections from HBV-ACLF

The hallmark of ACLF is the presence of sinusoidal thrombosis and associated hepatocellular necrosis, these phenomena are accompanied by the expression of virusinduced FGL2 in the cells of the sinusoidal lining. The FGL2 protein has the capacity to activate the coagulation cascades directly, a function that has been described as procoagulant activity (PCA) [4]. In this study, FGL2 expression was found in these HBV-ACLF samples, and immunofluorescence double staining indicated that $\mathrm{CK}-18^{+}$ epithelial cells, $\mathrm{CD}^{+} 8^{+}$macrophages and $\mathrm{CD} 31^{+}$endothelial cells are the major source of this protein. The expression of BTLA/HVEM and FGL2 in HBV-ACLF sections was further measured. The results demonstrated that both BTLA and HVEM were co-expressed with FGL2 (Figure 6). Our combined results revealed that both BTLA and HVEM were enriched in HBV-ACLF patients, suggesting that these molecules can be used as biomarkers for the pathological diagnosis of HBV-ACLF.

\section{Discussion}

The BTLA/HVEM inhibitory pathway appears to be particularly important for balancing immune response and 

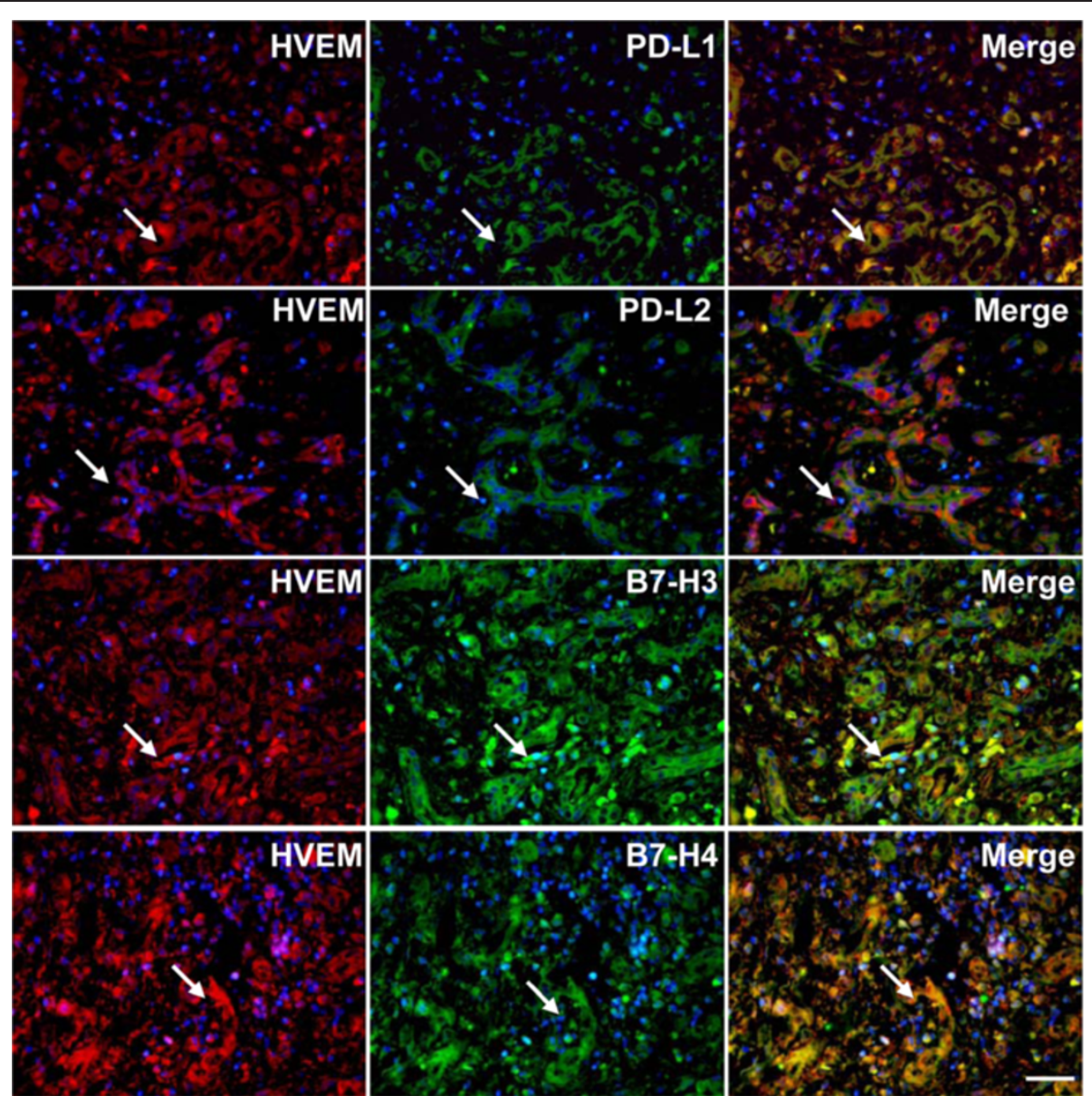

Figure 5 The relationship between the expression of BTLA/HVEM and certain members of the B7 superfamily in liver tissue sections from patients with HBV-related acute-on-chronic liver failure (HBV-ACLF). Immunofluorescence double staining indicated that several novel members of the B7 superfamily, including PD-L1, PD-L2, B7-H3 and B7-H4, were co-expressed with HVEM. The arrows indicate positive cells. The nuclei were stained with DAPI, and the scale bar indicates $20 \mu \mathrm{m}$.

tolerance in autoimmune diseases, tumor development, transplantation, allergy and, in particular, the pathogenesis of viral infection [16-18]. Recent studies have suggested that the BTLA/HVEM interaction functionally regulates $\mathrm{CMV}$-specific $\mathrm{T}$ cell functions [9]. Our previous work has also demonstrated that BTLA signaling can maintain the survival of macrophages that are infected with MHV-3, resulting in virus distribution and tissue damage following MHV-3 infection [13]. ACLF is a severe clinical syndrome characterized by coagulopathy, jaundice and hepatic encephalopathy $[19,20]$. Most ACLF cases in China are associated with HBV infection. In this study, we extended our research and examined the expression of BTLA/HVEM in four cases of HBV-ACLF patients. Immunohistochemical analysis revealed that the expression of BTLA was dramatically enhanced in liver samples from these HBV-ACLF patients, and the positive cells were distributed throughout the entire tissue. However, no positive cells were observed in the liver tissue of $\mathrm{CHB}$ patients or healthy individuals. The phenotypes of $\mathrm{BTLA}^{+}$cells were further analyzed by immunofluorescence double staining, and the results demonstrated that BTLA was expressed on CK- $18^{+}$epithelial cells, $\mathrm{CD} 31^{+}$endothelial cells, CD68 ${ }^{+}$ macrophages, $\mathrm{CD}^{2} 6^{+} \mathrm{NK}$ cells, $\mathrm{CD} 16^{+}$monocytes, $\mathrm{CD}^{+}$ $\mathrm{CD}^{+} \mathrm{T}$ cells, and Foxp3 ${ }^{+}$regulatory $\mathrm{T}$ cells (Tregs). Although the limitation of HBV-ACLF cases was included here, the expression of BTLA was observed on all of these four cases and these data strongly indicates that BTLA may actively participate in regulating the pathogenesis of this disease.

HVEM, the ligand of BTLA, is a novel member of the TNF receptor superfamily, and its mRNA is broadly expressed in both lymphoid and non-lymphoid tissues [18]. The expression and distribution of HVEM in liver biopsies from HBV and HBV-ACLF patients has not previously been reported. In this study, our data indicated that HVEM was found in sections from HBV-ACLF and 

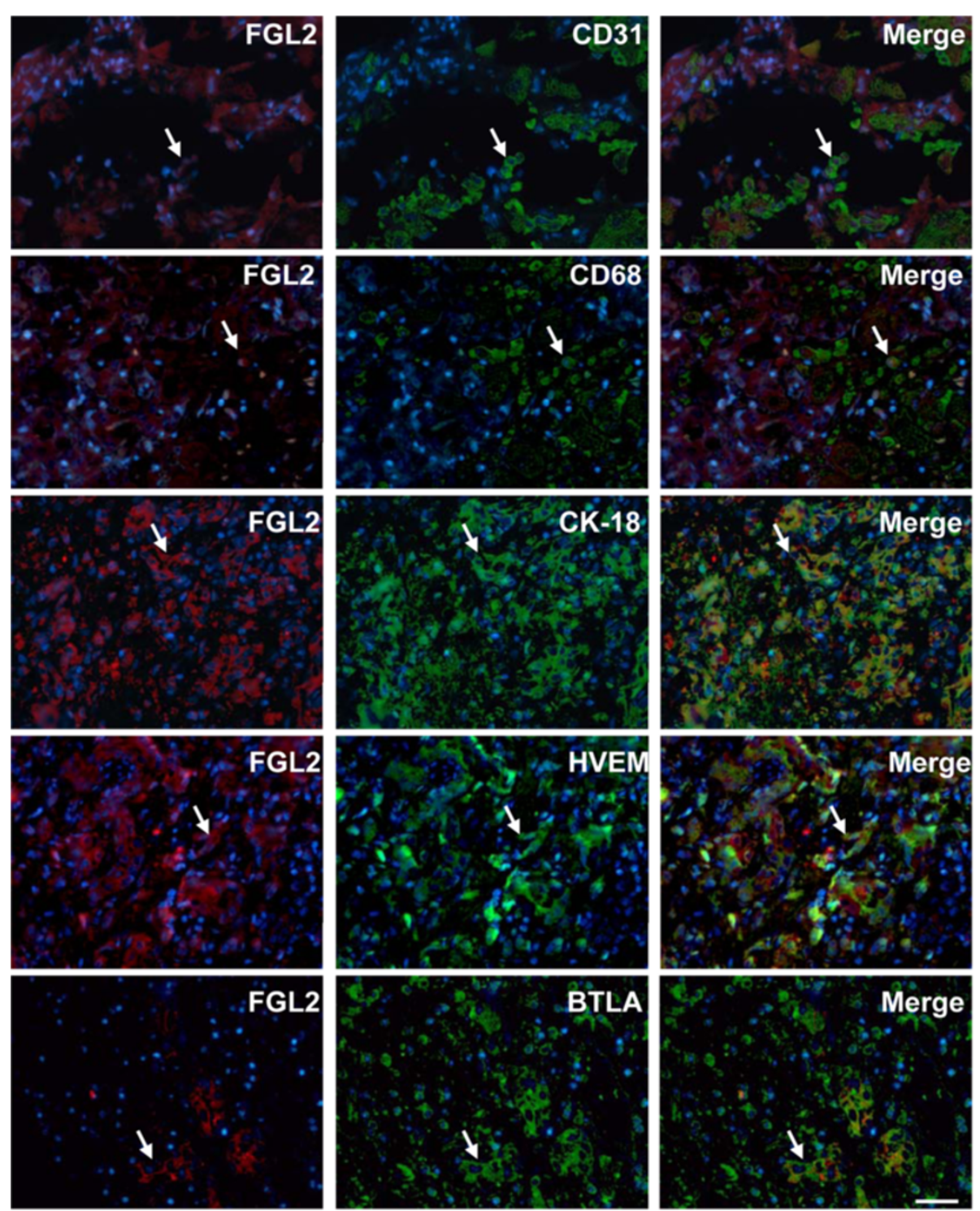

Figure 6 The relationship between BTLA-HVEM and fibrinogen-like protein 2 (FGL2) expression in sections from patients with HBVrelated acute-on-chronic liver failure (HBV-ACLF). Immunofluorescence double staining demonstrated that FGL2 expression was observed in $\mathrm{CD} 31^{+}$endothelial cells, CD68 ${ }^{+}$macrophages, and CK-18 ${ }^{+}$bile ducts of HBV-ACLF patients. Moreover, BTLA and HVEM were co-expressed with FGL2. The arrows indicate positive cells. The nuclei were stained with DAPI, and the scale bar indicates $20 \mu \mathrm{m}$.

was restricted to the damaged bile ducts and certain infiltrating cells, whereas HVEM was absent in sections from normal individuals. Assessments using immunofluorescence double staining further determined that HVEM was expressed on CK- $18^{+}$epithelial cells and $\mathrm{CD}^{+} 8^{+}$macrophages but completely absent on $\mathrm{CD} 31^{+}$ endothelial cells, $\mathrm{CD} 16^{+}$monocytes, and $\mathrm{CD}^{+} \mathrm{CD}^{+} \mathrm{T}$ cells. These results demonstrated that HVEM was induced in certain antigen-presenting cells (APCs) in these patients.

Our previous work has revealed that the expression of several members of the B7 superfamily, including PD-L1 (B7-H1), PD-L2 (B7-DC), B7-H3 and B7-H4, was found in liver sections from HBV-ACLF [14,15]. PD-L1 and PD-L2 are two immunoregulatory molecules belonging to the $\mathrm{B} 7$ superfamily that were identified as ligands for PD-1. The binding of PD-L1 or PD-L2 with PD-1 activates a signaling pathway that inhibits $T$ cell responses $[21,22]$. B7-H3 is a type I transmembrane protein that is expressed on activated macrophages, dendritic cells, monocytes and several types of cells in nonlymphoid tissues [23]. B7-H3 promotes the proliferation, cytotoxicity and IFN- $\gamma$ production of $\mathrm{T}$ cells by crosslinking with its hypothetical receptor and triggering the receptor-mediated expression of myeloid cell-like transcript 2 (TLT-2) on activated T cells [23,24]. B7-H4 is a 
GPI-linked protein, and mRNA encoding B7-H4 is widely distributed in murine and human peripheral tissues $[25,26]$. B7- $\mathrm{H} 4^{-1-}$ mice exhibited augmented Th1 responses and displayed lowered parasite burdens upon Leishmania major and Listeria monocytogenes infection compared with wild type mice, suggesting an inhibitory role for $\mathrm{B} 7-\mathrm{H} 4$ in $\mathrm{T}$ cell responses $[27,28]$. In this study, we also detected the relationships between HVEM and these members of the B7 superfamily in sections from HBV-ACLF, and the study results demonstrated that HVEM was co-expressed with all of these molecules.

FGL2 is a critical molecule that promotes fibrinogen deposition, which, in turn, activates the coagulation cascades and thereby induces PCA, causing hepatocellular necrosis during the development of fulminant virus hepatitis [2]. High levels of FGL2 ${ }^{+}$cells that are distributed throughout the liver have also been observed in HBV-ALCF patients. Phenotypic analyses indicated that the expression of FGL2 was mainly localized to infiltrating $\mathrm{CD} 8^{+}$macrophages, $\mathrm{CK}-18^{+}$bile ducts, and $\mathrm{CD} 31^{+}$ capillaries. Moreover, both BTLA and HVEM were coexpressed with FGL2, as assessed by immunofluorescence double staining.

To the best of our knowledge, this report is the first to investigate the expression of BTLA and its ligand, HVEM, in liver sections from HBV-ACLF patients. Our results indicate that these molecules might be useful diagnostic biomarkers for HBV-ACLF, and an understanding of the functional roles of these molecules could aid in the development of novel strategies for disease diagnosis or immunotherapy.

\section{Competing interests}

None of the authors have any conflicts of interest related to this manuscript.

\section{Authors' contributions}

HX: Immunohistochemistry; DC: Immunofluoresence double staining; GG: Samples collections and H\&E staining; ZR: Immunofluoresence double staining; YW: Revised the manuscript and experimental design; YC: Writing this manuscript and experimental design. All authors read and approved the final manuscript.

\section{Acknowledgments}

This work was supported by grants from the National Natural Science Foundation of China (NSFC No. 81171585, 30872374 and 61141012) and Natural Science Foundation of Chongqing City (No CSTC2011BB5037).

\footnotetext{
Author details

${ }^{1}$ Institute of Immunology, PLA, Third Military Medical University, Chongqing 400038, People's Republic of China. ${ }^{2}$ Undergraduate Administration Office, Third Military Medical University, Chongqing 400038, People's Republic of China. ${ }^{3}$ Department of Emergency, South-West Hospital, PLA, Third Military Medical University, Chongqing 400038, People's Republic of China.

${ }^{4}$ Department of Oncology, South-West Hospital, PLA, Third Military Medical University, Chongqing 400038, People's Republic of China.
}

Received: 28 September 2012 Accepted: 11 October 2012 Published: 15 October 2012

\section{References}

1. Stravitz RT, Kramer DJ: Management of acute liver failure. Nat Rev Gastroenterol Hepatol 2009, 6:542-553.

2. Liu Q, Liu Z, Wang T, et al: Characteristics of acute and sub-acute liver failure in China: nomination, classification and interval. $J$ Gastroen Hepatol 2007, 22:2101-2106

3. Liu M, Chan C, McGilvray l, et al: Fulminant viral hepatitis: molecular and cellular basis, and clinical implications. Expert Rev Mol Med 2001, 2001:1-19.

4. Marsden PA, Ning Q, Fung L, et al: The Fgl2/fibroleukin prothrombinase contributes to immunologically mediated thrombosis in experimental and human viral hepatitis. J Clin Invest 2003, 112:58-66.

5. Watanabe N, Gavrieli M, Sedy JR, et al: BTLA is a lymphocyte inhibitory receptor with similarities to CTLA-4 and PD-1. Nat Immuno 2003, 4:670-679.

6. Deppong $C$, Juehne TI, Hurchla $M$, et al: Cutting edge: B and T lymphocyte attenuator and programmed death receptor-1 inhibitory receptors are required for termination of acute allergic airway inflammation. J Immunol 2006, 176:3909-3913.

7. Oya Y, Watanabe N, Owada T, et al: Development of autoimmune hepatitis-like disease and production of autoantibodies to nuclear antigens in mice lacking B and T lymphocyte attenuator. Arthritis Rheum 2008, 58:2498-2510.

8. Tao R, Wang L, Han R, et al: Differential effects of B and T lymphocyte attenuator and programmed death-1 on acceptance of partially versus fully MHC-mismatched cardiac allografts. J Immunol 2005, 175:5774-5782.

9. Serriari NE, Gondois-Rey F, Guillaume $Y$, et al: B and T lymphocyte attenuator is highly expressed on CMV-specific T cells during infection and regulates their function. J Immunol 2010, 185:3140-3148.

10. Sun Y, Brown NK, Ruddy MJ, et al: B and T lymphocyte attenuator tempers early infection immunity. J Immunol 2009, 183:1946-1951.

11. Sedy JR, Gavrieli M, Potter KG, et al: B and T lymphocyte attenuator regulates $T$ cell activation through interaction with herpesvirus entry mediator. Nat Immunol 2005, 6:90-98.

12. Gavrieli M, Watanabe N, Loftin SK, et al: Characterization of phosphotyrosine binding motifs in the cytoplasmic domain of B and $T$ lymphocyte attenuator required for association with protein tyrosine phosphatases SHP-1 and SHP-2. Biochem Biophys Res Commun 2003, 312:1236-1243.

13. Yang C, Chen Y, Guo G, et al: Expression of B and T lymphocyte attenuator (BTLA) in macrophages contributes to the fulminant hepatitis caused by murine hepatitis virus strain-3. Gut 2012, Jun 9. [Epub ahead of print]

14. Cao D, Xu H, Guo G, et al: Intrahepatic Expression of Programmed Death1 and its Ligands in Patients with HBV-Related Acute-on-Chronic Liver Failure. Inflammation 2012, Aug 16. [Epub ahead of print].

15. Guo G, Cao D, Xu H, et al: The characteristic expression of B7-H3 and B7$\mathrm{H} 4$ in liver biopsies from patients with HBV-related acute-on-chronic liver failure. Pathology Int 2012, 62:665-674.

16. Murphy TL, Murphy KM: Slow down and survive: Enigmatic immunoregulation by BTLA and HVEM. Annu Rev Immunol 2010, 28:389-411.

17. Derré $L$, Rivals JP, Jandus $C$, et al: BTLA mediates inhibition of human tumor-specific CD8+ T cells that can be partially reversed by vaccination. J Clin Invest 2010, 120:157-167.

18. Pasero C, Speiser DE, Derré L, et al: The HVEM network: new directions in targeting novel costimulatory/co-inhibitory molecules for cancer therapy. Curr Opin Pharmacol 2012, 12:478-485.

19. Nascimento C, Bottino A, Nogueira C, et al: Analysis of morphological variables and arterialization in the differential diagnosis of hepatic nodules in explanted cirrhotic livers. Diagn Pathol 2007, 2:51.

20. Das $P$, Jain D, Das A: A retrospective autopsy study of histopathologic spectrum and etiologic trend of fulminant hepatic failure from north India. Diagn Pathol 2007, 2:27.

21. Dong H, Zhu G, Tamada K, et al: B7-H1, a third member of the B7 family, co-stimulates T-cell proliferation and interleukin-10 secretion. Nat Med 1999, 5:1365-1369.

22. Latchman $Y$, Wood CR, Chernova T, et al: PD-L2 is a second ligand for PD-1 and inhibits T cell activation. Nat Immuno 2001, 2:261-268.

23. Chapoval Al, Ni J, Lau JS, et al: B7-H3: A costimulatory molecule for T cell activation and IFN-gamma production. Nat Immunol 2001, 2:269-274. 
24. Hashiguchi M, Kobori $H$, Ritprajak $P$, et al: Triggering receptor expressed on myeloid cell-like transcript 2 (TLT-2) is a counterreceptor for B7-H3 and enhances T cell responses. Proc Natl Acad Sci USA 2008, 105:10495-10500.

25. Sica GL, Choi IH, Zhu G, et al: B7-H4, a molecule of the B7 family, negatively regulates T cell immunity. Immunity 2003, 18:849-861.

26. Prasad DV, Richards S, Mai XM, et al: B7S1, a novel B7 family member that negatively regulates T cell activation. Immunity 2003, 18:863-873.

27. Suh WK, Wang S, Duncan GS, et al: Generation and characterization of B7-H4/B7S1/B7x-deficient mice. Mol Cell Biol 2006, 26:6403-6411.

28. Zhu G, Augustine MM, Azuma T, et al: B7-H4-deficient mice display augmented neutrophil-mediated innate immunity. Blood 2009, 113:1759-1767.

doi:10.1186/1746-1596-7-142

Cite this article as:Xu et al:: The Intrahepatic Expression and Distribution of BTLA and its Ligand HVEM in patients with HBV-related acute-on-chronic liver failure. Diagnostic Pathology 2012 7:142.

\section{Submit your next manuscript to BioMed Central and take full advantage of:}

- Convenient online submission

- Thorough peer review

- No space constraints or color figure charges

- Immediate publication on acceptance

- Inclusion in PubMed, CAS, Scopus and Google Scholar

- Research which is freely available for redistribution 